\title{
Einige Bemerkungen über die Existenz orientierter Mannigfaltigkeiten mit vorgeschriebener mittlerer Krümmungsform
}

F. DUZNAR and M. FUCHS

Given an integral $m$-current $T_{0}$ in $\mathbb{R}^{m+k}$ and a tensor $H$ of type $(m, 1)$ on $\mathbb{R}^{m+k}$ with values orthogonal to each of its arguments we prove under suitable smallness conditions the existence of an integral $m$-current $T$ with boundary $\partial T=\partial T_{0}$ having prescribed mean curvature form $H$.

Key words: Integral currents, generalized mean curvature, small H-surfaces

AMS subject classification: $49 \mathrm{~F} 20,49 \mathrm{~F} 22,53 \wedge 10$

\section{Einführung}

In dieser Arbeit diskutieren wir die Frage nach der Existenz (orientierter) $m-$ dimensionaler Mannigfaltigkeiten $\Sigma$ in $\boldsymbol{R}^{m+k}$, wobei sowohl der Rand $\partial \Sigma=\Gamma$ als auch die mittlere Krümmung vorgegeben sein sollen. Zunächst ist jedoch zu klären, was man im Fall beliebiger Dimension und Kodimension unter dem Begriff der mittleren Krümmung zu verstehen hat. Bezeichnet etwa $\underline{\underline{H}}(x)$ den differentialgeometrischen Krümmungsvektor (= Spur der zweiten Fundamentalform) von $\Sigma$ im Punkt $x$, so erscheint es naheliegend, auf $\boldsymbol{R}^{\mathbf{m}+k}$ ein Vektorfeld $\mathcal{X}$ vorzuschreiben und $\Sigma$ in der Weise zu konstruieren, daB einerseits $\partial \Sigma=\Gamma$ und andererseits $\underline{H}(x)=\mathcal{X}$ erfüllt wird. Daß ein solches Vorgehen in der Regel scheitern muß, erkennt man unschwer bereits für Kodimension $k=1$ und der Wahl $\mathcal{X}(x)=\eta(x) e_{m+1}$ mit einer skalaren Funktion $\eta$ : hat nämlich $\Sigma$ in jedem Punkt $x$ die mittlere Krümmung $\underline{\underline{H}}(x)=\eta(x) e_{m+1}$, so folgt $T_{x} \Sigma=\boldsymbol{R}^{m} \times\{0\}$, d. h. $\Sigma$ ist eben und der mittlere Krümmungsvektor identisch 0 . $\mathrm{Zu}$ einer sinnvollen Begriffsbildung gelangt man dann, wenn man die mittlere Krümmung definiert als eine vektorwertige Funktion $H(x, E)$, die vom FuBpunkt $x \in \boldsymbol{R}^{m+k}$ und den $m$-dimensionalen Ebenen $E$ abhängt mit der zusätzlichen Eigenschaft

$$
H(x, E) \in E^{\perp} \text {. }
$$

Mit dieser Interpretation ist eine Mannigfaltigkeit $\Sigma$ Lösung des Problems, wenn neben $\partial \Sigma=\Gamma$ die Beziehung

$$
\underline{\underline{H}}(x)=\bar{H}\left(x, T_{x} \Sigma\right)
$$


in jedem Punkt $x \in \Sigma$ erfüllt ist. Mit Hilfe des Divergenzsatzes [16] läßt sich (1.1) äquivalent formulieren als

$$
\int_{\Sigma}\left\{\operatorname{div}_{\Sigma} X+X \cdot H\left(\cdot, T_{x} \Sigma\right)\right\} d \mathcal{H}^{m}=0
$$

für alle Vektorfelder $X \in C_{0}^{1}\left(\boldsymbol{R}^{m+k}, \boldsymbol{R}^{m+k}\right), \operatorname{spt}(X) \cap \Gamma=\emptyset$. Hierbei steht $\mathcal{H}^{m}$ für das $m$-dimensionale Hausdorff $\mathrm{MaB}$ im $\boldsymbol{R}^{m+k}$ (für die Definition sei auf $[6,2.10 .2$ ] verwiesen).

Um (1.2) als Euler-Gleichung eines Variationsfunktionals zu erkennen, schränken wir die Struktur von $H$ etwas ein und verlangen

$$
H \in C_{0}^{1}\left(\boldsymbol{R}^{m+k}, \bigwedge^{m}\left(\boldsymbol{R}^{m+k}, \boldsymbol{R}^{m+k}\right)\right)
$$

mit

$$
H(x, \xi) \in \operatorname{Span}\left[\xi_{1}, \ldots, \xi_{m}\right]^{\perp}
$$

für alle einfachen $m$-Vektoren $\xi=\xi_{1} \wedge \ldots \wedge \xi_{m}$. Die mittlere Krümmungsform $H(x, \cdot): \bigwedge_{m} \boldsymbol{R}^{m+k} \rightarrow \boldsymbol{R}^{m+k}$ ist also für jeden Basispunkt $x$ eine lineare Abbildung auf den $m$-Vektoren in $\boldsymbol{R}^{m+k}$ mit Werten in $\boldsymbol{R}^{m+k}$, die im Sinne von (1.3) auf ihren Argumenten senkrecht steht. Gulliver [7] folgend definieren wir die zu $H$ gehörende skalare mittlere Krümmungsform $h$ durch

$$
h_{x}\left(\xi_{0} \wedge \ldots \wedge \xi_{m}\right):=\xi_{0} \cdot H\left(x, \xi_{1} \wedge \ldots \wedge \xi_{m}\right)
$$

Ist $h$ geschlossen, also $h=d \alpha$ für eine $m$-Form $\alpha$, so besitzt unser Problem Variationsstruktur: Bezeichnet $\llbracket \Gamma \rrbracket$ den zu $\Gamma$ gehörigen $(m-1)$-Strom (wir setzen $\Gamma$ als orientiert voraus) und ist $T=\underline{\underline{\tau}}(M, \theta, \vec{T})$ ein ganzer $m$-Strom mit $\partial T=$ $\llbracket \Gamma \rrbracket$ (man vergleiche Abschnitt 2 für Notationen), so ist die erste Variation des Funktionals

$$
\mathbf{G}(T):=\mathbf{M}(T)+T(\alpha)
$$

in Richtung eines Vektorfeldes $X$ (wie in (1.2)) gegeben durch

$$
\langle\delta \mathbf{G}(T), X\rangle=\int_{M}\left\{\operatorname{div}_{M} X+X \cdot H(\cdot, \vec{T})\right\} d \mu_{T},
$$

$\mu_{T}=\mathcal{H}^{m} L \theta$, und motiviert durch (1.2) definieren wir: der ganze Strom $T$ (mit $\partial T=\llbracket \Gamma \rrbracket)$ hat vorgeschriebene mittlere Krümmungsform $H$, falls

$$
\langle\delta \mathrm{G}(T), \cdot\rangle \equiv 0
$$

gilt, und lokal in der Nähe eines regulären Punktes $x$ aus dem Träger von $T$ bedeutet dies, daß der geometrische mittlere Krümmungsvektor $\underline{\underline{H}}(x)$ der unterliegenden Mannigfaltigkeit gegeben ist durch $\underline{\underline{H}}(x)=H(x, \vec{T}(x))$. Man beachte, daB die mittlere Krümmungsform eine von der Orientierung abhängige Größe ist, d. h. der Strom $-T$ hat mittlere Krümmungsform $-H$, der mittlere Krümmungsvektor wird hierdurch natürlich nicht beeinflußt. 
Zur Lösung von (1.4) bietet es sich an, das Funktional $\mathbf{G}$ in geeigneten Klassen ganzer $m$-Ströme zu minimieren. Wie in [7] bemerkt ist dies im Falle einer globalen Schranke

$$
|\alpha| \leq 1-\varepsilon
$$

mit $\varepsilon>0$ ohne Schwierigkeiten möglich. Denkt man andererseits aber an konstante Krümmungsformen $H(x, \xi)=H(\xi)$, so ist (1.5) eine viel zu starke Forderung. In den Arbeiten [3-5] haben wir (1.5) durch geometrisch sinnvollere Bedingungen ersetzt und anstelle des Anteils $T(\alpha)$ im Funktional $\mathbf{G}(T)$ das sogenannte $h$-Volumen diskutiert und unter anderem Existenzsätze unter Einbeziehung von Almgren's Optimaler Isoperimetrischen Ungleichung [1] bewiesen.

In der vorliegenden Note beschreiten wir den Mittelweg, wobei wir uns an der aus dem parametrischen Kontext bekannten Konstruktion der "kleinen Lösung" orientieren, vgl. $[8-15,17,18]$ für eine Beschreibung der parametrischen Methoden.

Angenommen die Randmannigfaltigkeit $\Gamma$ ist kompakt und in einer offenen Kugel $B_{R}$ in $\boldsymbol{R}^{m+k}$ enthalten. Verlangt man

$$
\left|h_{x}\right| \leq \frac{m+1-\varepsilon}{R}
$$

mit $\varepsilon>0$ für alle $x \in \overline{B_{R}}$, so gibt es eine Stammform $\alpha$ mit

$$
\left|\alpha_{x}\right|<1 \quad \forall x \in \overline{B_{R}},
$$

und es liegt nahe, $\mathbf{G}$ auf den Strömen mit Träger in $\overline{B_{R}}$ zu minimieren, was nach (1.7) möglich ist. Ergänzt man (1.6) noch durch

$$
\left|h_{x}\right| \leq \frac{m-\varepsilon}{R} \quad \text { auf } \partial B_{R},
$$

so gilt ein Maximum-Prinzip, d. h. die Minimalstelle $T$ hat positiven Abstand $z u$ $\partial B_{R}$ und man bekommt die Euler-Gleichung (1.4). Somit lautet unser Hauptergebnis:

Unter den Bedingungen (1.6), (1.8) und für orientierte Ränder $\Gamma$ mit $\operatorname{dist}\left(\Gamma, \partial B_{R}\right)>0$ gibt es eine (verallgemeinerte) orientierte $m$-dimensionale Mannigfaltigkeit mit Rand $\Gamma$ und vorgeschriebener mittlerer Krümmungsform $H$.

Es sei bemerkt, daß entsprechende Aussagen möglich sind, wenn statt Kugeln andere strikt konvexe Körper betrachtet werden. Allerdings erscheint dann die Suche nach einer Stammform $\alpha$ mit geeigneter Kleinheitsbedingung wenig aussichtsreich. In diesem Zusammenhang liefert die Analyse des $h$-Volumens auf sytematischerem Weg Existenzsätze. 


\section{Notationen, Formulierung des Variationsproblems}

Seien $m \geq 2, k \geq 1$ ganze Zahlen, $B$ bezeichne die abgeschlossene Einheitskugel mit Mittelpunkt 0 in $\boldsymbol{R}^{m+k}$. Die Funktion $H: \boldsymbol{R}^{m+k} \rightarrow \bigwedge^{m}\left(\boldsymbol{R}^{m+k}, \boldsymbol{R}^{m+k}\right)$ sei von der Klasse $C^{1}$ und habe die Eigenschaft

$$
H_{z}\left(v_{1} \wedge \ldots \wedge v_{m}\right) \in \operatorname{Span}\left[v_{1}, \ldots, v_{m}\right]^{\perp}
$$

für alle $z, v_{1}, \ldots, v_{m} \in \boldsymbol{R}^{m+k}$. Zu $H$ definieren wir die skalare Krümmungsform $h: \boldsymbol{R}^{m+k} \rightarrow \bigwedge^{m+1} \boldsymbol{R}^{m+k}$ durch

$$
h_{z}\left(v_{0} \wedge \ldots \wedge v_{m}\right):=v_{0} \cdot H_{z}\left(v_{1} \wedge \ldots \wedge v_{m}\right)
$$

und verlangen $d h=0$. Dann wird durch

$$
\left\langle\alpha(z), \xi_{1} \wedge \ldots \wedge \xi_{m}\right\rangle:=\int_{0}^{1} t^{m}\left\langle h(t z), z \wedge \xi_{1} \wedge \ldots \wedge \xi_{m}\right\rangle d t
$$

eine Stammform zu $h$ erklärt, d. h. es gilt $d \alpha=h$. Für Differentalformen $\omega: U \rightarrow$ $\wedge^{n} R^{m+k}, U$ offen in $\mathbb{R}^{m+k}, n \in N$, seien

$$
\left|\omega_{z}\right|:=\sup _{\tau_{1} \cdot \tau,=\delta_{1} j}\left|\left\langle\omega(z), \tau_{1} \wedge \ldots \wedge \tau_{n}\right\rangle\right|
$$

und

$$
|\omega|:=\sup _{z \in U}\left|\omega_{z}\right|,
$$

im Falle von vektorwertigen Formen ist der Absolutbetrag rechts in (2.1) als Euklidische Norm zu verstehen.

Wir erinnen kurz an einige Notationen aus der Geometrischen Maßtheorie (vgl. $[6,16]): T \in \mathcal{D}_{n}\left(\boldsymbol{R}^{m+k}\right), n \in N$, heißt rektifizierbarer $n-S t r a m$ mit ganzer Vielfachheit, falls gilt

$$
T(\omega)=\int_{M}\langle\omega, \zeta\rangle \theta d \mathcal{H}^{n}, \quad \omega \in \mathcal{D}^{n}\left(\boldsymbol{R}^{m+k}\right),
$$

mit einer abzählbar $n$-rektifizierbaren Menge $M \subset \boldsymbol{R}^{m+k}$, einer lokal $\mathcal{H}^{n}$-integrierbaren Dichtefunktion $\theta: M \rightarrow N_{0}$ und einer $\mathcal{H}^{n}$-meßbaren Funktion

$$
\zeta: M \rightarrow \bigwedge_{n}\left(\boldsymbol{R}^{m+k}\right),
$$

wobei $\zeta(x)$ in $\mathcal{H}^{n}$-fast allen Punkten $x \in M$ gegeben ist durch

$$
\zeta(x)=\tau_{1}(x) \wedge \ldots \wedge \tau_{n}(x)
$$

für eine Orthonormalbasis $\left\{\tau_{i}(x)\right\}$ des approximativen Tangentialraumes $T_{x} M$. Ist $\partial T$ rektifizierbarer $(n-1)$-Strom mit ganzzahliger Vielfachheit, so nennen wir $T$ einen ganzen Strom. Es sei

$$
\mathbf{I}^{n}:=\left\{T \in \mathcal{D}_{n}\left(\boldsymbol{R}^{m+k}\right): T \text { ist ganzer Strom, } \mathbf{M}(T)+\mathbf{M}(\partial T)<\infty\right\} .
$$


In diesem Zusammenhang sei darauf hingewiesen, daß die Masse $\mathbf{M}(T)$ eines rektifizierbaren $n$-Stromes mit ganzer Vielfach heit nicht von der speziellen Normierung (2.1), (2.2) der $n$-Formen abhängt.

Schließlich fixieren wir $T_{0} \in I^{m}$ (den Rand) und definieren

$$
\mathcal{C}:=\left\{T \in \mathrm{I}^{m}: \operatorname{spt}(T) \subset B, \partial T=\partial T_{0}\right\}
$$

und

$$
\mathbf{G}(T):=\mathbf{M}(T)+T(\alpha), \quad T \in \mathcal{C},
$$

wobei wir natürlich $\mathcal{C} \neq \emptyset$ annnehmen, was beispielsweise durch die Forderung $\operatorname{spt}\left(T_{0}\right) \subset B$ garantiert wird.

Satz 2.1: Das Variationsproblem

$$
\mathbf{G}(\cdot) \leadsto \operatorname{Min} \text { in } \mathcal{C}
$$

besitzt eine Lösung, vorausgesetzt man verlangt

$$
\sup _{z \in B}\left|H_{z}\right|<m+1
$$

Beweis: Es ist für $z \in B, \xi_{i} \cdot \xi_{j}=\delta_{i j}$

$$
\begin{aligned}
\left\langle\alpha(z), \xi_{1} \wedge \ldots \wedge \xi_{m}\right\rangle & \leq \int_{0}^{1} t^{m} d t \sup _{x \in B}\left|\left\langle h(x), x \wedge \xi_{1} \wedge \ldots \wedge \xi_{m}\right\rangle\right| \\
& =(m+1)^{-1} \sup _{x \in B}\left|\left\langle h(x), \Pi_{\xi}(x) \wedge \xi_{1} \wedge \ldots \wedge \xi_{m}\right\rangle\right|
\end{aligned}
$$

wenn $\Pi_{\xi}(x)$ den Anteil von $x$ senkrecht $z$ u Span $\left[\xi_{1}, \ldots, \xi_{m}\right]$ bezeichnet. Also gilt

$$
\left\langle\alpha(z), \xi_{1} \wedge \ldots \wedge \xi_{m}\right\rangle \leq(m+1)^{-1} \sup _{x \in B}|x|\left|h_{x}\right| .
$$

Mit (2.3) folgt

$$
\sup _{z \in B}\left|\alpha_{z}\right| \leq 1-\varepsilon
$$

für ein $\varepsilon \in(0,1)$, also

$$
\mathbf{G}(T) \geq \varepsilon \mathbf{M}(T),
$$

so daß eine G-Minimalfolge $\left\{T_{n}\right\}$ in $\mathcal{C}$ eine Teilfolge besitzt, die in $\mathcal{D}_{m}\left(\boldsymbol{R}^{m+k}\right)$ gegen $T \in \mathcal{C}$ konvergiert. Da die $T_{n}$ alle Träger in $B$ haben, gilt trivialerweise $T_{n}(\alpha) \rightarrow T(\alpha)$. Zusammen mit der Unterhalbstetigkeit der Masse $\mathbf{M}$ erhalten wir

$$
\mathbf{G}(T) \leq \liminf _{n \rightarrow \infty} \mathbf{G}\left(T_{n}\right)
$$

Folglich ist $T$ eine gesuchte Minimalstelle. 


\section{Ein Maximumprinzip}

Der Beweis des folgenden Satzes orientiert sich an [8].

Satz 3.1: Für die mittlere Krümmungsform $H$ gelte

$$
\left|H_{z}\right|<m+1 \quad \text { auf } B
$$

und

$$
\left|H_{2}\right|<m \quad \text { auf } \partial B .
$$

Außerdem sei der Träger von $\partial T_{0}$ kompakt im Inneren von $B$ enthalten. Ist dann $T$ die in Satz 2.1 erklärte Lösung des Variationsproblems

$$
\mathbf{G}(\cdot) \sim \operatorname{Min} \text { in } \mathcal{C},
$$

so folgt für alle genügend kleinen $r>0$

$$
\mathcal{H}^{m}\left(\left\{z \in \boldsymbol{R}^{m+k}:|z| \geq 1-r\right\} \cap \operatorname{spt}(T)\right)=0,
$$

d. h. T hat kompakten Träger im Inneren von $B$,

Beweis: Sei $\operatorname{dist}\left(\partial T_{0}, \partial B\right)>\varrho_{0} ;$ für $0 \leq t \leq \varrho_{0}$ und $z \in B$ definiert man

$$
F(t, z):= \begin{cases}z, & |z| \leq 1-t \\ (1-t) z /|z|, & |z| \geq 1-t\end{cases}
$$

Dann gilt mit beliebigem $0<\varrho<\varrho_{0}$, daB $F(\varrho, \cdot)_{\#} T \in \mathcal{C}$ und

$$
\begin{aligned}
\mathbf{G}( & \left.F(\varrho, \cdot)_{\#} T\right)-\mathbf{G}(T) \\
= & \mathbf{M}\left(F(\varrho, \cdot)_{\#} T\right)-\mathbf{M}(T)+\left(F(\varrho, \cdot)_{\#} T\right)(\alpha)-T(\alpha) \\
= & \int_{|z| \geq 1-\varrho}\left\{\left|D F(\varrho, \cdot)_{\#} \vec{T}\right|-1\right\} d \mu_{T} \\
& +\int_{|z| \geq 1-\varrho}\left\{\left\langle\alpha(F(\varrho, \cdot)), D F(\varrho, \cdot)_{\#} \vec{T}\right\rangle-\langle\alpha, \vec{T}\rangle\right\} d \mu_{T} .
\end{aligned}
$$

Für $z$ aus dem Integrationsbereich ist

$$
\left|D F(\varrho, z)_{\#} \vec{T}(z)\right| \leq(1-\varrho)^{m}|z|^{-m}
$$

und (mit $G(z):=z /|z|)$

$$
\begin{aligned}
& \left\langle\alpha(F(\varrho, z)), D F(\varrho, z)_{\#} \vec{T}(z)\right\rangle-\langle\alpha(z), \vec{T}(z)\rangle \\
& \quad=\int_{1-|z|}^{e} \frac{\partial}{\partial s}\left\langle\alpha(F(s, z)), D F(s, z)_{\#} \vec{T}(z)\right\rangle d s \\
& \quad=\int_{1-|z|}^{e} \frac{\partial}{\partial s}\left\{\int_{0}^{1} t^{m}\left\langle h(t F(s, z)), F(s, z) \wedge D F(s, z)_{\#} \vec{T}(z)\right\rangle d t\right\} d s
\end{aligned}
$$




$$
\begin{aligned}
& =\int_{1-|z|}^{e} \frac{\partial}{\partial s}\left\{\int_{0}^{1} t^{m}\left\langle h(t(1-s) G(z)), G(z) \wedge D G(z)_{\#} \vec{T}(z)\right\rangle(1-s)^{m+1} d t\right\} d s \\
& =\int_{1-|z|}^{e} \frac{\partial}{\partial s}\left\{\int_{0}^{1-s} u^{m}\left\langle h(u G(z)), G(z) \wedge D G(z)_{\#} \vec{T}(z)\right\rangle d u\right\} d s \\
& =-\int_{1-|z|}^{e}(1-s)^{m}\left\langle h((1-s) G(z)), G(z) \wedge D G(z)_{\#} \vec{T}(z)\right\rangle d s \\
& \leq|z|^{-m} \sup _{|z| \geq 1-e}\left|h_{x}\right| \int_{1-|z|}^{e}(1-s)^{m} d s .
\end{aligned}
$$

Kombiniert man dies mit (3.3), so folgt insgesamt

$$
\begin{aligned}
\mathbf{G} & \left(F(\varrho, \cdot)_{\#} T\right)-\mathbf{G}(T) \\
& \leq \int_{|z| \geq 1-\varrho}|z|^{-m}\left\{\left((1-\varrho)^{m}-|z|^{m}\right)+c(\varrho) \int_{1-|z|}^{e}(1-s)^{m} d s\right\} d \mu_{T}(z) \\
& =\int_{|z| \geq 1-e}|z|^{-m} \int_{1-|z|}^{e}\left(\frac{d}{d t}(1-t)^{m}+c(\varrho)(1-t)^{m}\right) d t d \mu_{T}(z) \\
& =\int_{|z| \geq 1-e}|z|^{-m} \int_{1-|z|}^{e} m(1-t)^{m-1}\left(\frac{c(\varrho)}{m}(1-t)-1\right) d t d \mu_{T}(z)
\end{aligned}
$$

wobei wir $c(\varrho):=\sup _{1 \geq|x| \geq 1-\varrho}\left|h_{x}\right|$ gesetzt haben. Nach (3.2) gibt es ein $\varepsilon>0$ mit $c(\varrho) \leq m(1-\varepsilon)$ für alle $\varrho \ll 1$, d. h.

$$
\mathrm{G}\left(F(\varrho, \cdot)_{\#} T\right)-\mathrm{G}(T) \leq \varepsilon \int_{|z| \geq 1-e}|z|^{-m}\left((1-\varrho)^{m}-|z|^{m}\right) d \mu_{T}(z)
$$

und im Falle

$$
\mu_{T}(\{z \in B:|z| \geq 1-e\})>0
$$

ist die rechte Seite der vorstehenden Ungleichung kleiner als Null, was der Minimalität von $T$ widerspricht.

\section{Die Euler-Gleichung}

In diesem Abschnitt zeigen wir, daß die Lösungen unserer Variationsaufgabe die vorgeschriebene mittlere Krümmungsform $H$ besitzen.

Satz 4.1: Der Krümmungsvektor $H$ erfülle

$$
\left|H_{z}\right|<m+1 \quad \text { auf } B
$$

und

$$
\left|H_{z}\right|<m \quad \text { auf } \partial B .
$$

$T$ bezeichne die Lösung von

$$
\mathbf{G}(\cdot) \leadsto \operatorname{Min} \text { in } \mathcal{C} \text {. }
$$


Dann gilt für alle Vektorfelder $X \in C_{0}^{1}\left(\boldsymbol{R}^{m+k}, \boldsymbol{R}^{m+k}\right), \operatorname{spt}(X) \cap \operatorname{spt}(\partial T)=\emptyset$, die Eulergleichung $\left(T=\underline{\underline{\tau}}(M, \theta, \vec{T}), \mu_{T}=\mathcal{H}^{m}\llcorner\theta)\right.$

$$
\int_{M}\left\{\operatorname{div}_{M} X+X \cdot H(\cdot, \vec{T})\right\} d \mu_{T}=0
$$

Korollar 4.1: Für Punkte $x \in \operatorname{spt}(T) \backslash \operatorname{spt}(\partial T)$ gilt der Allard'sche Regularitätssatz.

Bemerkung: Wie in [3, Theorem 7.1] gezeigt, bleibt die Aussage von Satz 4.1 richtig, wenn (4.1) durch $\left|H_{z}\right| \leq m$ auf $\partial B$ ersetzt wird. Allerdings sind dann kompliziertere Argumente nötig.

Beweis: Für $X$ wie im Satz und $|t|$ genügend klein und

$$
\varphi(x, t):=x+X(x)
$$

folgt aus dem Maximum-Prinzip $\varphi(\cdot, t)_{\#} T \in \mathcal{C}$, also

$$
\begin{aligned}
\frac{d}{d t} \mathrm{G}\left(\varphi(\cdot, t)_{\#} T\right)_{\mid t=0} \\
=\int_{M} \operatorname{div}_{M} X d \mu_{T}+\frac{d}{d t}\left(\varphi(\cdot, t)_{\#} T\right)(\alpha)_{\mid t=0} \\
=\int_{M} \operatorname{div}_{M} X d \mu_{T}+\frac{d}{d t}\left\{\int_{M}\left\langle\alpha(\varphi(\cdot, t)), D \varphi(\cdot, t)_{\#} \vec{T}\right\rangle d \mu_{T}\right\}_{\mid t=0} \\
=\int_{M} \operatorname{div}_{M} X d \mu_{T}+\int_{M}\left\langle\alpha^{\prime}(z)(X(z)), \vec{T}(z)\right\rangle d \mu_{T}(z) \\
\quad+\int_{M}\left\langle\alpha, \sum_{i=1}^{m} \tau_{1} \wedge \ldots \wedge \partial_{\tau_{i}} X \wedge \ldots \wedge \tau_{m}\right\rangle d \mu_{T},
\end{aligned}
$$

wobei $\alpha^{\prime}(z)$ die totale Ableitung von $\alpha$ in $z$ bedeutet. Bei festem $z$ gilt die Beziehung

$$
\left\langle d \alpha(z), \xi_{1} \wedge \ldots \xi_{m+1}\right\rangle=\sum_{i=1}^{m+1}(-1)^{i-1}\left\langle\alpha^{\prime}(z) \xi_{i}, \xi_{1} \wedge \ldots \wedge \xi_{i-1} \wedge \xi_{i+1} \wedge \ldots \wedge \xi_{m+1}\right\rangle
$$

und mit $\xi:=X(z) \wedge \vec{T}(z)$ erhält man sofort

$$
\begin{aligned}
& \langle d \alpha(z), X(z) \wedge \vec{T}(z)\rangle=\left\langle\alpha^{\prime}(z)(X(z)), \vec{T}(z)\right\rangle \\
& \quad+\sum_{j=1}^{m}(-1)^{j}\left\langle\alpha^{\prime}(z) \tau_{j}(z), X(z) \wedge \tau_{1}(z) \wedge \ldots \wedge \tau_{j-1}(z) \wedge \tau_{j+1}(z) \wedge \ldots \wedge \tau_{m}(z)\right\rangle .
\end{aligned}
$$

Gleichzeitig ist für $\xi \in \Lambda_{m}\left(\boldsymbol{R}^{m+k}\right), \xi=\xi_{1} \wedge \ldots \wedge \xi_{m}$,

$$
\begin{aligned}
& \sum_{i=1}^{m}\left\langle\alpha(z), \xi_{1} \wedge \ldots \wedge \partial_{\xi_{1}} X(z) \wedge \ldots \wedge \xi_{m}\right\rangle \\
& \quad=\sum_{i=1}^{m} \partial_{\xi_{1}}\left\langle\alpha(z), \xi_{1} \wedge \ldots \wedge X(z) \wedge \ldots \wedge \xi_{m}\right\rangle+\sum_{i=1}^{m}\left(\alpha^{\prime}(z) \xi_{i}, \xi_{1} \wedge \ldots \wedge X(z) \wedge \ldots \wedge \xi_{m}\right\rangle \\
& \quad=-\left\langle d \lambda(z), \xi_{1} \wedge \ldots \wedge \xi_{m}\right\rangle+\sum_{i=1}^{m}(-1)^{i}\left\langle\alpha^{\prime}(z) \xi_{i}, X(z) \wedge \xi_{1} \wedge \ldots \xi_{i-1} \wedge \xi_{i+1} \wedge \ldots \wedge \xi_{m}\right\rangle
\end{aligned}
$$


wobei wir

$$
\left\langle\lambda(z), \eta_{1} \wedge \ldots \eta_{m-1}\right\rangle:=\left\langle\alpha(z), X(z) \wedge \eta_{1} \wedge \ldots \eta_{m-1}\right\rangle
$$

definiert haben. Man beachte hierbei die Formel

$$
\begin{aligned}
\left\langle d \lambda(z), \xi_{1} \wedge \ldots \wedge \xi_{m}\right\rangle & =\sum_{l=1}^{m}(-1)^{l-1}\left\langle\lambda^{\prime}(z) \xi_{l}, \xi_{1} \wedge \ldots \wedge \xi_{l-1} \wedge \xi_{l+1} \wedge \ldots \wedge \xi_{m}\right\rangle \\
& =\sum_{l=1}^{m}(-1)^{l-1} \partial_{\xi_{l}}\left(\lambda(z), \xi_{1} \wedge \ldots \wedge \xi_{l-1} \wedge \xi_{l+1} \wedge \ldots \wedge \xi_{m}\right\rangle
\end{aligned}
$$

Insgesamt ergibt sich:

$$
\begin{aligned}
\int_{M}\left\langle\alpha^{\prime}(z) X, \vec{T}\right\rangle d \mu_{T} & +\int_{M}\left\langle\alpha, \sum_{i=1}^{m} \tau_{1} \wedge \ldots \wedge \partial_{\tau_{1}} X \wedge \ldots \tau_{m}\right\rangle d \mu_{T} \\
& =-\int_{M}\langle d \lambda, \vec{T}\rangle d \mu_{T}+\int_{M}\langle d \alpha, X \wedge \vec{T}\rangle d \mu_{T} \\
& =\int_{M}\langle d \alpha, X \wedge \vec{T}\rangle d \mu_{T} \\
& =\int_{M} X \cdot H(\cdot \vec{T}) d \mu_{T},
\end{aligned}
$$

denn $\lambda$ hat offenbar kompakten Träger disjunkt zu $\operatorname{spt}(\partial T)$. Dies beweist die Euler-Gleichung (4.3).

Für den Beweis des Korollars vergleiche man $[2,16]$.

\section{Literat urverzeichnis}

[1] F. J. Almgren, Optimal Isoperimetric Inequalities. Indisna Univ. Math. J. 35 (1986), 451-547

[2] W. K. Allard, On the first variation of a varifold. Ann. of Math. 95 (1972), 417-491.

[3] F. Duzaar, M. Fuchs, On the existence of integral currents with prescribed mean curvature vector. manus. math. 67 (1990), 41-67.

[4] F. Duzaar, M. Fuchs, Existence of area minimizing tangent cones of integral currents with prescribed mean curvature. SFB preprint no. 80, Universität Bonn, 1989.

[5] F. Duzaar, M. Fuchs, On Integral Currents with Constant Mean Curvature. Erscheint in Rend. Cont. Sem. Mat. Padova (1991).

[6] $H$. Federer, Geometric measure theory. Berlin-Heidelberg-New York 1969.

[7] R. Gulliver, Necessary conditions for submanifolds and currents with prescribed mean curvature vector. Seminar on minimal submanifolds, ed. E. Bombieri, Princeton 1983.

[8] R. Gulliver, J. Spruck, Existence theorems for parametric surfaces of prescribed mean curvature. Indiana Univ. Math. J. 22 (1972), 445-472.

[9] E. Heinz, Über die Existenz einer Fläche vorgeschriebener mittlerer Krümmung bei vorgegebener Berandung. Math. Ann. 127 (1954), 258-287. 
[10] S. Hildebrandt, Einige Bemerkungen über Flächen beschränkter mittlerer Krümmung Math. Z. 115 (1970), 169-178.

[11] $S$. Hildebrandt, Über einen neuen Existenzsatz für Flächen vorgeschriebener mittlerer Krümmung. Math. Z. 119 (1971), 267-272.

[12] K. Steffen, Isoperimetric inequalities and the problem of Plateau. Math. Ann. 222 (1976), 97-144.

[13] $K$. Steffen, On the existence of surfaces with prescribed mean curvature and boundary. Math. Z. 146 (1976), 113-135.

[14] K. Steffen, Ein verbesserter Existenzsatz für Flächen konstanter mittlerer Krümmung. Manus. Math. 6 (1972), 105-139.

[15] K. Steffen, Flächen konstanter mittlerer Krümmung mit vorgegebenem Volumen oder Flächeninhalt. Arch. Rat. Mech. Analysis 49 (1972),99-128.

[16] L. Simon, Lectures on geometric measure theory. Proceedings C. M.A. 3, Canberra 1983.

[17] M. Struwe, Plateau's problem and the calculus of variations. Mathematical notes 35, Princeton University Press, 1988.

[18] H. Wente, An existence theorem for surfaces of constant mean curvature. J. Math. Analysis Appl. 26 (1969), 318-344.

Manuskripteingang: 29, 05, 1990

Dr. Frank Duzaar

Mathematisches Institut

der Heinrich-Heine-Universität

Universitätsstr. 1

D-4000 Düsseldorf
Prof. Dr. Martin Fuchs

TH Darmstadt

Fachbereich 4/ AG 6

SchloBgartenstr. 7

D 6100 Darmstadt 